\title{
The future looks brighter after 25 years of retinal gene therapy
}

\author{
Alberto Auricchio ${ }^{1}$, Alexander J Smith ${ }^{2}$ and Robin R Ali ${ }^{2}$
}

1. TIGEM

Naples, Italy

2. Department of Genetics

UCL Institute of Ophthalmology

London EC1V 9EL

\begin{abstract}
The first report of in vivo gene delivery to the retina dates back to 1987 when a retroviral vector was injected intraocularly in newborn mice. Later came the observation that retinal cells could be successfully transduced using adenoviral and, then, adeno-associated and lentiviral vectors. By 2000, it had became clear that the eye, compared with other organs and tissues, provides a number of advantages for in vivo gene therapy with regard to safety, efficacy and route to clinical application. This has prompted the development of many successful proof-of-concept studies in animal models. The demonstration that sight could be restored in a large animal model with a congenital form of blindness was a major landmark that opened the door to the first-in-human trials for recessively inherited blinding conditions. With these first human studies demonstrating safety and some efficacy, retinal gene therapy has now come of age. The rapid clinical development of retinal gene therapy has highlighted various new challenges, such as management of inflammation and development of new vectors that allow optimization. The treatment of patients with advanced photoreceptor degeneration or dominantly inherited retinal dystrophies and the delivery of large genes, yet given the progress over the last 25 years, the next decade promises a bright future for retinal gene therapy.
\end{abstract}

\section{Text}

In 1987 the group of Connie Cepko used intraocular delivery of retroviral vectors to label rodent retinal cell progenitors with reporter genes. ${ }^{1}$ Although it was not for therapeutic purposes, this study was the first report of in vivo gene delivery to the retina. In the last twenty five years, however, there has been a rapid expansion of ocular gene delivery for therapeutic purposes. There have been significant contributions from many different groups, many of them European. In 1994 the groups of Beverly Davidson ${ }^{2}$ and Stephen Jones ${ }^{3}$ observed efficient and effective delivery to retinal pigment epithelium (RPE) cells with an adenoviral vector. Efficient transduction of photoreceptors, the main target cells for gene therapy of inherited retinal degeneration, had to wait for the development of what has since become the most effective and widely used vector for retinal gene therapy, the adeno-associated viral vector (AAV). Subretinal administration of AAV serotype 2 in adult rodents resulted in efficient photoreceptor transduction in addition to RPE. 4,5 Around the same time, administration of newly developed HIV1-based lentiviral vectors to the retina of 
newborn mice resulted in transduction of RPE and photoreceptor cells. ${ }^{6}$ However, even in newborn animals the levels of photoreceptor transduction achieved did not reach the levels that could be achieved using AAV-based vectors, leaving the latter as the vector of choice for treating most retinal disorders. Subsequently, many more naturally occurring $7,8,9$ or engineered ${ }^{10,11}$ AAV serotypes have been assessed for their ability to transduce the retina with the aim of identifying serotypes with advantageous properties: different cell tropisms, a greater ability to penetrate the inner retina or to mediate higher levels of transgene expression. In general, most AAV vectors are able to mediate efficient and life-long transduction of the neuroretina.

From these initial experiments with vectors encoding reporter genes it was apparent that the retina offered several advantages in terms of in vivo gene delivery when compared to other tissues. Whilst intraocular administrations are technically challenging, they allow precise exposure of the tissue to vector. The amount of vector required to obtain substantial retinal exposure is limited, minimizing both risks of toxicity and the challenge of large-scale viral vector production. The retina is relatively immune-privileged, and the presence of two eyes allows the delivery/treatment of one leaving the contralateral eye as an intra-individual control. Furthermore, a number of small and large animal models of inherited retinal diseases (IRDs) were available to test safety and efficacy of retinal gene therapy. These have been instrumental for the development of the many proof-of-concept studies that have been reported since the late 1990s. There are dozens of examples of rodent models in which retinal structure and function have been substantially improved by subretinal administration of viral vectors, most commonly AAV. ${ }^{12}$ Perhaps the first clear proof-of-concept study was carried out by Robin Ali and colleagues who used subretinal delivery of an AAV2 vector to deliver a gene encoding the structural protein peripherin/rds to restore photoreceptor structure and function .13 14

In the early 2000s, came the observation that efficacy of retinal gene therapy in small animals could be replicated in larger species. These studies not only demonstrated that scale-up of retinal gene therapy was feasible but also that restoration of vision was was evident by observing the behavior of treated animals, and thus even the lay public could appreciate the beneficial effect of retinal gene therapy in a visually impaired dogs. The pivotal study was performed by a group of US investigators, including Bill Hauswirth, Sam Jacobson and Gus Aguirre and co-ordinated by Jean Bennett. They demonstrated that a single subretinal administration of an AAV2 vector carrying an RPE65 gene resulted in long term restoration of night vision in Briard dogs affected by Leber congenital amaurosis type 2 (LCA2) caused by RPE65 deficiency. ${ }^{15}$

In 2005 Hurwitz and colleagues treated retinoblastoma in children using intraocular injection of an adenoviral vector carrying a suicide gene. ${ }^{16}$ The procedure was well tolerated, and in several cases resolution of the intravitreous tumor seeds was observed. This study represents the first example of an ocular gene therapy clinical trial involving a viral vector. It was soon followed by several other ocular gene therapy trials. The dramatic improvement in function 
obtained in the Briard dogs paved the way for the development of the first clinical trials of gene therapy for an inherited retinal dystrophy. In 2007-2008 there were three independent clinical trials of gene therapy for LCA2, involving subretinal administration of AAV2 vectors carrying RPE65. The trials, two in the US and one in the UK, showed that AAV-mediated gene therapy could be safe and effective, with improvements in visual acuity, pupillary reflex, light sensitivity and ability to navigate in dim light.17,18,19 Therapeutic benefit was observed several weeks after injection and at least some benefit was observed in some individuals even 3 years after treatment. However, two of the trials, reported a decline in the level of visual improvement was not sustained long term 20,21 highlighting the potential need for either higher levels of transgene expression or for a wider retinal transduction. This has prompted the development of a new trial for LCA2 using AAV5, a AAV serotype that provides a more efficient retinal transduction than AAV2, in combination with an optimized transgene expression cassette in order to provide higher levels of RPE65 (NTC02946879, Table I). ${ }^{22}$ At the same time a phase III clinical trial has been developed (NCT00999609, Table I) using an original AAV2 vector with the objective of collecting sufficient data to obtain market authorization. ${ }^{23}$ Whatever, the eventual outcome of the current efforts to develop an effective gene therapy for LCA2, the positive results obtained by different groups has facilitated a rapid expansion in the number of clinical trials of gene therapy for other IRDs. These include conditions such as choroideremia, ${ }^{24}$ Leber optic neuropathy, ${ }^{25}$ or Stargardt disease, ${ }^{26}$ among others (Table I) and also common complex diseases, such as age-related macular degeneration (AMD) (Table I). ${ }^{27,28}$

The contribution of European academic groups and companies to the retinal gene therapy clinical trials has been substantial. In the UK, trials of therapies for LCA2 (NCT 00643747 and 02781480), X-linked RP (NCT03252847) and CNGB3 achromatopsia (NCT03001310) have been developed by UCL/Moorfields Eye Hospital and MeiraGTx; trials for chroideremia (NCT01461213) and X-linked RP (NCT03116113) by The University of Oxford and NightStarX and trials for Usher type 1B (NCT01505062), Stargardt disease (NCT01367444) and AMD (NCT01301443) by Oxford Biomedica. In France a trial for Leber hereditary optic neuropathy (NCT02064569) has been developed by the Institut de la Vision, and GenSight in Paris as well as a trial for LCA2 using AAV4 (NCT01496040) by The University of Nantes. In Germany, a trial for CNGA3 achromatopsia (NCT02610582) has been developed by groups at the University Hospital Tübingen and the Ludwig-Maximilians-University Munich.

Table I. Retinal gene therapy trials (updated on June 7th, 2017, from Clinicaltrials.gov)

\begin{tabular}{|c|c|c|c|}
\hline Disease & Vector & $\begin{array}{l}\text { Number } \\
\text { (NCT) }\end{array}$ & Notes \\
\hline \multirow{4}{*}{ Achromatopsia } & AAV8-hCARp.hCNGB3 & 03001310 & Phase 1/2, Recruiting \\
\hline & AAV2tYF-CNGA3 & 02935517 & Phase $1 / 2$, Not yet Recruiting \\
\hline & AAV2tYF-CNGB3 & 02599922 & Phase $1 / 2$, Recruiting \\
\hline & AAV8-hCNGA3 & 02610582 & Phase $1 / 2$, Recruiting \\
\hline \multirow[t]{5}{*}{ Choroideremia } & AAV2-hCHM & 02341807 & Phase 1/2, Recruiting \\
\hline & AAV2-REP1 & 02407678 & Phase 2, Recruiting \\
\hline & AAV2-REP1 & 01461213 & Phase $1 / 2$, Active not recruiting \\
\hline & AAV2-REP1 & 02077361 & Phase $1 / 2$, Active not recruiting \\
\hline & AAV2-REP1 & 02671539 & Phase 2, Active not recruiting \\
\hline
\end{tabular}




\begin{tabular}{|c|c|c|c|}
\hline \multirow{10}{*}{ Leber Congenital Amaurosis 2} & AAV2-hRPE65v2 & 01208389 & Phase 1/2 (follow-on), Active not recruiting \\
\hline & AAV5-OPTIRPE65 & 02946879 & LTFU (Phase 1/2), Recruiting \\
\hline & AAV2-hRPE65v2-301 & 00999609 & Phase 3 , Active not recruiting \\
\hline & AAV2-hRPE65v2-101 & 00516477 & Phase 1/2, Active not recruiting \\
\hline & AAV5-OPTIRPE65 & 02781480 & Phase 1, Recruiting \\
\hline & AAV2.hRPE65p.hRPE65 & 00643747 & Phase $1 / 2$, Completed \\
\hline & AAV2-hRPE65 & 00749957 & Phase 1/2, Active not recruiting \\
\hline & AAV2-hRPE65 & 00481546 & Phase 1, Active not recruiting \\
\hline & AAV2-hRPE65 & 00821340 & Phase 1, Unknown \\
\hline & AAV4-hRPE65 & 01496040 & Phase 1/2, Completed \\
\hline \multirow{6}{*}{ Leber Hereditary Optic Neuropathy } & scAAV2-P1ND4v2 & 02161380 & Phase 1, Recruiting \\
\hline & AAV2-ND4 & 02652780 & Phase 3, Active not recruiting \\
\hline & AAV2-ND4 & 02652767 & Phase 3, Recruiting \\
\hline & AAV2-ND4 & 03153293 & Phase 2/3, Recruiting \\
\hline & AAV2-ND4 & 01267422 & Phase 1/2, Completed \\
\hline & AAV2-ND4 & 02064569 & Phase 1/2, Active not recruiting \\
\hline \multirow{6}{*}{$\begin{array}{l}\text { Neovascular/Age-related Macular } \\
\text { Degeneration }\end{array}$} & AAV.sFlt1 & 01494805 & Phase 2a, Unknown \\
\hline & AAV2-sFLT01 & 01024998 & Phase 1, Active not recruiting \\
\hline & AAV8-AntiVEGF & 03066258 & Phase 1, Recruiting \\
\hline & $A A V-C D 59$ & 03144999 & Phase 1, Recruiting \\
\hline & LV (Retinostat) & 01301443 & Phase 1, Completed \\
\hline & LV (Retinostat) & 01678872 & Phase 1 FU, Recruiting (by invitation) \\
\hline Retinitis Pigmentosa & AAV-ChR2 & 02556736 & Phase 1/2, Recruiting \\
\hline Stargardt Disease & LV (SAR422459) & 01367444 & Phase 1/2, Recruiting (by Invitation) \\
\hline \multirow[t]{2}{*}{ Usher Syndrome Type 1B } & LV (Ushstat) & 01505062 & Phase 1/2, Recruiting \\
\hline & LV (Ushstat) & 02065011 & Phase 1/2, Recruiting (by Invitation) \\
\hline \multirow[t]{2}{*}{ X-linked Retinitis Pigmentosa } & AAV-RPGR & 03116113 & Phase 1/2, Recruiting \\
\hline & AAV2/5-hRK.RPGR & 03252847 & Phase 1/2, Recruiting \\
\hline \multirow[t]{2}{*}{ X-linked Retinoschisis } & $A A V-R S 1$ & 02317887 & Phase 1/2, Recruiting \\
\hline & AAV2tYF-hRS1 & 02416622 & Phase 1/2, Recruiting \\
\hline
\end{tabular}

The rapid clinical development of gene therapy, including retinal gene therapy, suggests that the use of nucleic acids as drugs is finally coming of age. The multitude of trials, using various vectors, promoters, administration routes and doses will establish the basic toolkit for clinical gene therapy of the retina. Over the next couple of year, by assessing and comparing immune responses, vector shedding and effects of intraocular vector administration on retinal structure and function the safety and efficacy of particular vectors, routes of administration and doses will be determined. Whilst initial development in mice and dogs has provided a sound starting point for these trials, it is becoming increasingly clear that pre-clinical data alone does not necessarily enable effective translation of a protocol from animals to humans and for many therapies further optimization is likely to be required at the clinical trial stage.

As shown in Table I, the majority of the early trials have been in rare inherited disorders, but gene therapy can also be used for more common complex disorders. The condition most widely studied with regard to gene therapy has been neovascular AMD, since existing, but expensive pharmacological treatments had already validated VEGF as an effective target. ${ }^{29}$ For that reason, the failure of the first anti-VEGF gene therapy using AAV-sFlt1 was a disappointment. ${ }^{27} \mathrm{~A}$ positive outcome should have been achievable, but questionable clinical trial design and surgical issues have led to an ambiguous conclusion regarding therapeutic efficacy. This set back should not prevent further development of this area. The potential for major benefits to patients and socioeconomic benefits due to a single administration of a vector to target neovascularization versus repeated administration of a pharmacological agent has led to the development 
of various other anti-angiogenic gene therapy approaches and the initiation of a number of early phase clinical trials (table I).(refs) 28,30

Recent and ongoing retinal gene therapy trials are also highlighting some of the challenges that the field has to overcome to make this approach widely used. One is that subretinal administration of vector may induce irreversible damage to a tissue that has been thinned by many years of progressive degeneration. In this situation, less invasive intravitreal injections would be preferable. Yet the current generation of viral vectors do not cross the retinal layers from the vitreous to the photoreceptors. To solve this issue, the groups of Schaffer and Flannery set up a strategy based on in vivo directed evolution of libraries of thousands of AAV capsid variants that were administered to the vitreous of mice. The variants able to cross the retinal layers from the vitreous were isolated from photoreceptors. Enrichment in one variant, AAV7M8, occurred through repeated cycles of intravitreal injections, followed by photoreceptor harvesting and AAV capsid sequence isolation. ${ }^{10,31}$ As a result, AAV7M8 shows unprecedented ability to transduce mouse outer retina from the vitreous. ${ }^{31}$ Confirming this ability in retinas of larger species with thicker physical barriers than mice is ongoing and the first results look promising. ${ }^{32}$ AAV7M8, or other variants with similar properties ${ }^{33,34}$ may one day allow to avoid subretinal injection of therapeutic viral vectors in patients with IRDs.

One particular challenge to achieving a substantial clinical impact in the field of retinal gene therapy is the large number of genes involved in IRDs (https://sph.uth.edu/retnet/). Development of a generic gene therapy for photoreceptor cell loss has thus been a longstanding goal. Animal studies have shown that gene transfer of neurotrophic molecules, including CNTF, 35 GDNF36,37 and $\mathrm{bFGF}, 38$ or anti-apoptotic proteins, such as XIAP, ${ }^{39}$ to the retina can prolong the photoreceptor cell survival although this does not always lead to preservation of remaining vision. ${ }^{40}$ To date there have been no clinical trials of neurotrophic or anti-apoptotic gene therapy, perhaps because implantation of encapsulated cells secreting CNTF directly into the vitreous showed a more pronounced loss of light sensitivity in the treated eye, that reversed after removal of the implant. No long-term evidence of improved vision or preserved retinal structure was found. ${ }^{41}$

One-third of RP patients with a recognizable pattern of inheritance are affected by dominant RP of which many are due to toxic gain-of-function mutations (https://sph.uth.edu/retnet/). Among these are RHO P23H and P347S mutations for which there are useful transgenic mouse models ${ }^{42,43}$. Reducing levels of the toxic product rather than adding a correct copy of the gene is expected to provide significant benefits. The first attempts in this direction used allelespecific ribozymes to knock-down $\mathrm{RHO}$ P23H expression. ${ }^{44}$ The discovery of RNA interference in mammalian cells led to the development by the group of Jane Farrar, Trinity College Dublin, and others of AAV-delivered short hairpin RNAs (shRNAs) which would knock-down both the wild-type and mutant RHO allele coupled with replacement of a $R H O$ cDNA resistant to the shRNA. ${ }^{45} \mathrm{~A}$ different approach tuses artificial transcriptional repressors developed in the lab of Enrico M. Surace that silence $R H O$ expression, again in an allele-independent 
manner thus requiring supply of a $R H O$ copy that is resistant to silencing. ${ }^{46}$ Although pre-clinical results are promising and there are efforts to further develop these strategies into the clinic, one has to consider that knocking down RHO expression might convert a mild dominant RP into more severe recessive conditions that require expression of high levels of rhodopsin, the most abundant protein in photoreceptors.

The last decade has also witnessed the development of new tools for genome editing that allow efficient engineering of genes in situ. Among these tools are Znfinger (ZF) nucleases, TALE nucleases and, more recently, those based on the CRISPR-associated RNA-guided Cas9.47 Each of these systems allows precise induction of double strand breaks at specific genomic loci that can be repaired either by non-homologous end-joining, potentially knocking out specific dominant mutant alleles or by homology-directed repair that corrects the gene defect using a donor DNA template. The versatility and efficacy of these systems has increased over the years to levels that now reach therapeutic efficacy in the retina of animal models. ${ }^{48}$ However, off-target mutagenesis and the effects of prolonged expression of nucleases to terminally differentiated photoreceptors need to be carefully evaluated before clinical application.

For a substantial group of IRD patients the 'standard' gene therapy protocols described above will not be feasible. They are the advanced cases where most if not all photoreceptors have been lost to the degenerative process. Optogenetics may offer an alternative therapeutic strategy to regenerative medicine for those severely damaged retinas with no remaining photoreceptors. Gene transfer of bacterial opsins, ${ }^{49,50}$ or even rhodopsin, ${ }^{51}$ to post-photoreceptor neurons like bipolar or retinal ganglion cells has the potential to convert them in light sensing neurons. Although a clinical trial of optogenetic gene therapy is being prepared based on Channelrhodopsin2, the current generation of halorhodopsins or channelrhodopsins still requires abnormally high light intensities to be effectively excited. ${ }^{52,53}$ The optogenetics field however is constantly developing. Improvement of optogenetic tools for ocular therapeutics can be achieved by engineering of the channels to display optimised characteristics. Channel engineering has been used previously to improve their function as neuroscientific tools, e.g. sacrificing light sensitivity to achieve faster channel kinetics. ${ }^{54}$ For therapeutic use, such fast kinetics are not necessary and a channel with kinetics equivalent to or somewhat slower than cone phototransduction, is likely to display better light sensitivity. Moreover, using different vector serotypes it may be possible to transduce bipolar cells rather than ganglion cells and achieve more advanced intraretinal processing.

One important limitation of AAV vectors, which to date are the most effective platform for gene delivery to photoreceptor cells, are their cargo capacity that is limited to around $5 \mathrm{~kb}$ of DNA. This prevents their application to gene therapy of common and severe IRDs like Stargardt disease, Usher IB or LCA10 which are due to mutations in genes with a coding sequence larger than $5 \mathrm{~kb}$. To overcome this limitation, the labs of Shannon Boye, Alberto Auricchio and Robert McLaren, among others, have developed strategies based on dual AAV vectors each 
packaging one half of a large transgene expression cassette. Subretinal delivery of dual AAV vectors results in co-infection of RPE and photoreceptor cells followed by dual AAV genome recombination which reconstitutes expression of full length transcript and protein. This process can provide in therapeutic levels of transgene expression in mouse models of Stargardt disease or Usher IB, ${ }^{55}$ and opens the possibility to treat these conditions with AAV vectors.

Thirty years have passed since the first viral vectors were injected in developing retinas to label the various retinal cell types. Since then hundreds of patients have received intraocular injections of viral vectors. There have been few adverse effects and already some patients have signs of improved vision, an unprecedented observation in conditions where progression towards blindness has been always considered inevitable. Whilst the recent trials have highlighted new challenges and it is clear that further optimization and new approaches are still required before gene therapy is a routine treatment for the many individuals affected by retinal disorders, after 25 years of research involving many researchers around the world, including many European researchers, we are now seeing light at the end of the tunnel.

\section{Reference List}

1. Price, J, Turner, D, Cepko, C Lineage analysis in the vertebrate nervous system by retrovirus-mediated gene transfer. Proc Natl Acad Sci U S A 1987;84:156-160.

2. Li, T, Adamian, M, Roof, $\mathrm{D}$ et al. In vivo transfer of a reporter gene to the retina mediated by an adenoviral vector. Invest Ophthalmol Vis Sci 1994;35:2543-2549.

3. Jomary, C, Piper, TA, Dickson, G et al. Adenovirus-mediated gene transfer to murine retinal cells in vitro and in vivo. FEBS Lett 1994;347:117-122.

4. Ali, RR, Reichel, MB, Thrasher, AJ et al. Gene transfer into the mouse retina mediated by an adeno-associated viral vector. Hum Mol Genet 1996;5:591594.

5. Flannery, JG, Zolotukhin, S, Vaquero, MI et al. Efficient photoreceptortargeted gene expression in vivo by recombinant adeno-associated virus. Proc Natl Acad Sci U S A 1997;94:6916-6921.

6. Miyoshi, H, Takahashi, M, Gage, FH et al. Stable and efficient gene transfer into the retina using an HIV-based lentiviral vector. Proc Natl Acad Sci U S A 1997;94:10319-10323.

7. Auricchio, A Pseudotyped AAV vectors for constitutive and regulated gene expression in the eye. Vision Res 2003;43:913-918. 
8. Allocca, M, Mussolino, C, Garcia-Hoyos, M et al. Novel adeno-associated virus serotypes efficiently transduce murine photoreceptors. J Virol 2007;81:11372-11380.

9. Vandenberghe, LH, Bell, P, Maguire, AM et al. AAV9 targets cone photoreceptors in the nonhuman primate retina. PLoS One 2013;8:e53463

10. Klimczak, RR, Koerber, JT, Dalkara, D et al. A novel adeno-associated viral variant for efficient and selective intravitreal transduction of rat Muller cells. PLoS One 2009;4:e7467

11. Zinn, E, Pacouret, S, Khaychuk, V et al. In Silico Reconstruction of the Viral Evolutionary Lineage Yields a Potent Gene Therapy Vector. Cell Rep 2015;12:1056-1068.

12. Smith, AJ, Bainbridge, JW, Ali, RR Gene supplementation therapy for recessive forms of inherited retinal dystrophies. Gene Ther 2012;19:154161.

13. Ali, RR, Sarra, GM, Stephens, C et al. Restoration of photoreceptor ultrastructure and function in retinal degeneration slow mice by gene therapy. Nat Genet 2000;25:306-310.

14. Sarra, GM, Stephens, C, De Alwis, M et al. Gene replacement therapy in the retinal degeneration slow (rds) mouse: the effect on retinal degeneration following partial transduction of the retina. Hum Mol Genet 2001;10:23532361.

15. Acland, GM, Aguirre, GD, Ray, J et al. Gene therapy restores vision in a canine model of childhood blindness. Nat Genet 2001;28:92-95.

16. Chevez-Barrios, P, Chintagumpala, M, Mieler, $\mathrm{W}$ et al. Response of retinoblastoma with vitreous tumor seeding to adenovirus-mediated delivery of thymidine kinase followed by ganciclovir. J Clin Oncol 2005;23:7927-7935.

17. Bainbridge, JWB, Smith, AJ, Barker, SS et al. Effect of gene therapy on visual function in Leber's congenital amaurosis. N Engl J Med 2008;358:22312239.

18. Maguire, AM, Simonelli, F, Pierce, EA et al. Safety and efficacy of gene transfer for Leber's congenital amaurosis. N Engl J Med 2008;358:22402248.

19. Hauswirth, W, Aleman, TS, Kaushal, S et al. Phase I Trial of Leber Congenital Amaurosis due to RPE65 Mutations by Ocular Subretinal Injection of Adeno-Associated Virus Gene Vector: Short-Term Results. Hum Gene Ther 2008;19:979-990.

20. Cideciyan, AV, Jacobson, SG, Beltran, WA et al. Human retinal gene therapy for Leber congenital amaurosis shows advancing retinal degeneration 
despite enduring visual improvement. Proc Natl Acad Sci U S A 2013;110:E517-E525.

21. Bainbridge, JW, Mehat, MS, Sundaram, V et al. Long-term effect of gene therapy on Leber's congenital amaurosis. N Engl J Med 2015;372:18871897.

22. Georgiadis, A, Duran, Y, Ribeiro, J et al. Development of an optimized AAV2/5 gene therapy vector for Leber congenital amaurosis owing to defects in RPE65. Gene Ther 2016;23:857-862.

23. Russell, S, Bennett, J, Wellman, JA et al. Efficacy and safety of voretigene neparvovec (AAV2-hRPE65v2) in patients with RPE65-mediated inherited retinal dystrophy: a randomised, controlled, open-label, phase 3 trial. Lancet 2017

24. MacLaren, RE, Groppe, M, Barnard, AR et al. Retinal gene therapy in patients with choroideremia: initial findings from a phase $1 / 2$ clinical trial. Lancet 2014;383:1129-1137.

25. Feuer, WJ, Schiffman, JC, Davis, JL et al. Gene Therapy for Leber Hereditary Optic Neuropathy: Initial Results. Ophthalmology 2016;123:558-570.

26. Binley, K, Widdowson, PS, Kelleher, $\mathrm{M}$ et al. Safety and biodistribution of an equine infectious anemia virus-based gene therapy, RetinoStat((R)), for age-related macular degeneration. Hum Gene Ther 2012;23:980-991.

27. Constable, IJ, Pierce, CM, Lai, CM et al. Phase 2a Randomized Clinical Trial: Safety and Post Hoc Analysis of Subretinal rAAV.sFLT-1 for Wet Age-related Macular Degeneration. EBioMedicine 2016;14:168-175.

28. Heier, JS, Kherani, S, Desai, S et al. Intravitreous injection of AAV2-sFLT01 in patients with advanced neovascular age-related macular degeneration: a phase 1, open-label trial. Lancet 2017;390:50-61.

29. $\mathrm{Ba}, \mathrm{J}$, Peng, RS, $\mathrm{Xu}, \mathrm{D}$ et al. Intravitreal anti-VEGF injections for treating wet age-related macular degeneration: a systematic review and meta-analysis. Drug Des Devel Ther 2015;9:5397-5405.

30. Campochiaro, PA, Lauer, AK, Sohn, EH et al. Lentiviral Vector Gene Transfer of Endostatin/Angiostatin for Macular Degeneration (GEM) Study. Hum Gene Ther 2017;28:99-111.

31. Dalkara, D, Byrne, LC, Klimczak, RR et al. In vivo-directed evolution of a new adeno-associated virus for therapeutic outer retinal gene delivery from the vitreous. Sci Transl Med 2013;5:189ra76

32. Ramachandran, PS, Lee, V, Wei, Z et al. Evaluation of Dose and Safety of AAV7m8 and AAV8BP2 in the Non-Human Primate Retina. Hum Gene Ther 2017;28:154-167. 
33. Petrs-Silva, H, Dinculescu, A, Li, Q et al. Novel properties of tyrosine-mutant AAV2 vectors in the mouse retina. Mol Ther 2011;19:293-301.

34. Mowat, FM, Gornik, KR, Dinculescu, A et al. Tyrosine capsid-mutant AAV vectors for gene delivery to the canine retina from a subretinal or intravitreal approach. Gene Ther 2014;21:96-105.

35. Liang, FQ, Dejneka, NS, Cohen, DR et al. AAV-mediated delivery of ciliary neurotrophic factor prolongs photoreceptor survival in the rhodopsin knockout mouse. Mol Ther 2001;3:241-248.

36. McGee Sanftner, LH, Abel, H, Hauswirth, WW et al. Glial cell line derived neurotrophic factor delays photoreceptor degeneration in a transgenic rat model of retinitis pigmentosa. Mol Ther 2001;4:622-629.

37. Buch, PK, MacLaren, RE, Duran, $\mathrm{Y}$ et al. In Contrast to AAV-Mediated Cntf Expression, AAV-Mediated Gdnf Expression Enhances Gene Replacement Therapy in Rodent Models of Retinal Degeneration. Mol Ther 2006;14:700709.

38. Schuettauf, F, Vorwerk, C, Naskar, R et al. Adeno-associated viruses containing bFGF or BDNF are neuroprotective against excitotoxicity. Curr Eye Res 2004;29:379-386.

39. Yao, J, Feathers, KL, Khanna, H et al. XIAP therapy increases survival of transplanted rod precursors in a degenerating host retina. Invest Ophthalmol Vis Sci 2011;52:1567-1572.

40. Schlichtenbrede, FC, MacNeil, A, Bainbridge, JW et al. Intraocular gene delivery of ciliary neurotrophic factor results in significant loss of retinal function in normal mice and in the Prph2Rd2/Rd2 model of retinal degeneration. Gene Ther 2003;10:523-527.

41. Birch, DG, Bennett, LD, Duncan, JL et al. Long-term Follow-up of Patients With Retinitis Pigmentosa Receiving Intraocular Ciliary Neurotrophic Factor Implants. Am J Ophthalmol 2016;170:10-14.

42. Sakami, S, Kolesnikov, AV, Kefalov, VJ et al. P23H opsin knock-in mice reveal a novel step in retinal rod disc morphogenesis. Hum Mol Genet 2014;23:1723-1741.

43. Li, T, Snyder, WK, Olsson, JE et al. Transgenic mice carrying the dominant rhodopsin mutation P347S: evidence for defective vectorial transport of rhodopsin to the outer segments. Proc Natl Acad Sci U S A 1996;93:1417614181.

44. LaVail, MM, Yasumura, D, Matthes, MT et al. Ribozyme rescue of photoreceptor cells in $\mathrm{P} 23 \mathrm{H}$ transgenic rats: long-term survival and latestage therapy. Proc Natl Acad Sci U S A 2000;97:11488-11493. 
45. Millington-Ward, S, Chadderton, N, O'Reilly, M et al. Suppression and replacement gene therapy for autosomal dominant disease in a murine model of dominant retinitis pigmentosa. Mol Ther 2011;19:642-649.

46. Botta, S, Marrocco, E, de, PN et al. Rhodopsin targeted transcriptional silencing by DNA-binding. Elife 2016;5:e12242

47. Fernandez, A, Josa, S, Montoliu, L A history of genome editing in mammals. Mamm Genome 2017

48. Latella, MC, Di Salvo, MT, Cocchiarella, $\mathrm{F}$ et al. In vivo Editing of the Human Mutant Rhodopsin Gene by Electroporation of Plasmid-based CRISPR/Cas 9 in the Mouse Retina. Mol Ther Nucleic Acids 2016;5:e389

49. Bi, A, Cui, J, Ma, YP et al. Ectopic expression of a microbial-type rhodopsin restores visual responses in mice with photoreceptor degeneration. Neuron 2006;50:23-33.

50. Tomita, H, Sugano, E, Yawo, $\mathrm{H}$ et al. Restoration of visual response in aged dystrophic RCS rats using AAV-mediated channelopsin-2 gene transfer. Invest Ophthalmol Vis Sci 2007;48:3821-3826.

51. Cehajic-Kapetanovic, J, Eleftheriou, C, Allen, AE et al. Restoration of Vision with Ectopic Expression of Human Rod Opsin. Curr Biol 2015;25:21112122.

52. Busskamp, V, Duebel, J, Balya, D et al. Genetic reactivation of cone photoreceptors restores visual responses in retinitis pigmentosa. Science 2010;329:413-417.

53. Sengupta, A, Chaffiol, A, Mace, E et al. Red-shifted channelrhodopsin stimulation restores light responses in blind mice, macaque retina, and human retina. EMBO Mol Med 2016;8:1248-1264.

54. Hegemann, $\mathrm{P}$ and Moglich, A Channelrhodopsin engineering and exploration of new optogenetic tools. Nat Methods 2011;8:39-42.

55. Trapani, I, Colella, P, Sommella, A et al. Effective delivery of large genes to the retina by dual AAV vectors. EMBO Mol Med 2014;6:194-211. 